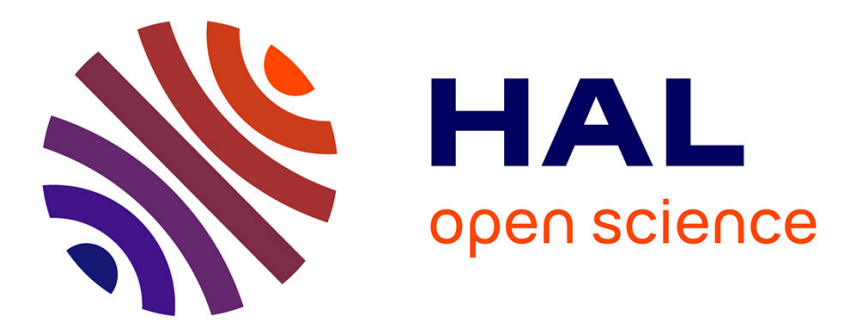

\title{
Metamorphosis-Induced Changes in the Coupling of Spinal Thoraco-Lumbar Motor Outputs During Swimming in Xenopus laevis
}

Anna Beyeler, Charles Métais, Denis Combes, John Simmers, Didier Le Ray

\section{To cite this version:}

Anna Beyeler, Charles Métais, Denis Combes, John Simmers, Didier Le Ray. Metamorphosis-Induced Changes in the Coupling of Spinal Thoraco-Lumbar Motor Outputs During Swimming in Xenopus laevis. Journal of Neurophysiology, 2008, 100 (3), pp.1372-1383. 10.1152/jn.00023.2008 . hal-02347096

\author{
HAL Id: hal-02347096 \\ https://hal.science/hal-02347096
}

Submitted on 7 Nov 2019

HAL is a multi-disciplinary open access archive for the deposit and dissemination of scientific research documents, whether they are published or not. The documents may come from teaching and research institutions in France or abroad, or from public or private research centers.
L'archive ouverte pluridisciplinaire HAL, est destinée au dépôt et à la diffusion de documents scientifiques de niveau recherche, publiés ou non, émanant des établissements d'enseignement et de recherche français ou étrangers, des laboratoires publics ou privés. 
Metamorphosis-induced changes in the coupling of spinal thoraco-lumbar motor outputs during swimming in Xenopus laevis.

Anna Beyeler, Charles Métais, Denis Combes, John Simmers \& Didier Le Ray.

Université de Bordeaux

Centre National de la Recherche Scientifique

Laboratoire Mouvement Adaptation Cognition (UMR 5227)

146, rue Léo Saignat

33076 Bordeaux

France

Running title: Lumbo-thoracic coupling during amphibian locomotion

Corresponding author: Didier Le Ray

didier.leray@u-bordeaux2.fr

Key words: Amphibian, motor networks, propriospinal pathways, development, locomotion, posture. 


\section{ABSTRACT}

Anuran metamorphosis includes a complete remodeling of the animal's biomechanical apparatus, requiring a corresponding functional reorganization of underlying central neural circuitry. This involves changes that must occur in the coordination between the motor outputs of different spinal segments in order to harmonize locomotor and postural functions as the limbs grow and the tail regresses. In pre-metamorphic Xenopus laevis tadpoles, axial motor output drives rostro-caudally propagating segmental myotomal contractions that generate propulsive body undulations. During metamorphosis, the anterior axial musculature of the tadpole progressively evolves into dorsal muscles in the post-metamorphic froglet, in which some of these back muscles lose their implicit locomotor function to serve exclusively in postural control in the adult. To understand how locomotor and postural systems interact during locomotion in juvenile Xenopus, we have investigated the coordination between postural back and hindlimb muscle activity during free forward swimming. Axial/dorsal muscles, which contract in bilateral alternation during undulatory swimming in pre-metamorphic tadpoles, change their left-right coordination to become activated in phase with bilaterally-synchronous hindlimb extensions in locomoting juveniles. Based on in vitro electrophysiological experiments as well as specific spinal lesions in vivo, a spinal cord region was delimited in which propriospinal interactions are directly responsible for the coordination between leg and back muscle contractions. Our findings therefore indicate that dynamic postural adjustments during adult Xenopus locomotion are mediated by local intraspinal pathways through which the lumbar generator for hindlimb propulsive kicking provides caudo-rostral commands to thoracic spinal circuitry controlling the dorsal trunk musculature. 


\section{INTRODUCTION}

Many behavioral tasks in invertebrates and vertebrates alike are driven by primary motor programs that arise from neuronal network assemblages within the central nervous system. Vertebrate locomotion is one of the best understood of such functions, the basic motor program for which being generated by local spinal networks that are controlled by higher centers in the brainstem and are continually shaped by sensory information from the periphery (Rossignol et al. 2006). Over the last thirty years, many studies on a variety of animal species have analyzed the functional organization of spinal and supra-spinal structures involved in the orchestration of rhythmic locomotor movements (for reviews, see Cazalets and Bertrand 2000; Grillner 2006; Kiehn 2006). In quadrupedal vertebrates, the locomotor programs for anterior and posterior limb movements are elaborated by so-called "central pattern generators" (CPGs; for reviews, see Grillner 1981; Kiehn 2006) that are located in the cervical (Ballion et al. 2001; Juvin et al. 2005) and lumbar spinal enlargements, respectively (Cazalets et al. 1995; Langlet et al. 2005). For meaningful locomotory behavior, however, musculature in other body regions such as the trunk and neck must be coordinated with limb movements to ensure postural stability during body displacements. Rhythmic activation of axial trunk muscles in time with appendicular locomotor activity has been reported in rat (Gramsbergen et al. 1999, Falgairolle and Cazalets 2007), cat (Koehler et al. 1984; Zomlefer et al. 1984) and humans (Thorstensson et al. 1982). However, although earlier experiments on decerebrate paralyzed cat suggested a propriospinal origin of coordinated limb-trunk muscle contractions (Koehler et al. 1984), the neural basis of such coupling and the extent to which it is governed by rhythmogenic spinal circuitry remains largely unknown (Falgairolle and Cazalets 2007). Similarly, little is known of 
the changing functional relationship that must occur between locomotory and postural control systems during post-natal development.

Metamorphosis from tadpole to frog in anuran amphibians like Xenopus laevis constitutes one of the most striking developmental transformations in biology, involving fundamental alterations in virtually all of the animal's physiological systems and body structure (for a review, see Shi 2000). One of the most dramatic changes occurs in the biomechanical apparatus, whereby the tail is resorbed and new limbs are formed as the organism changes its mode of locomotion from tail-based undulations in larvae to limbbased propulsion in the adult. In pre-metamorphic tadpoles, body displacement is driven by waves of bilaterally-alternating muscle contractions that are directed rostro-caudally along the body axis, whereas in post-metamorphic juveniles, bilaterally-synchronous hindlimb kicking is principally used to propel the animal forward. Previous studies strongly suggested that this metamorphosis-induced transition in locomotor strategy results principally from the gradual emergence of a lumbar CPG specifically dedicated to the control of the newly developed hindlimbs (Combes et al. 2004; Rauscent et al. 2007). However, the functional destiny of original axial motor circuitry that persists in the adult spinal cord remains to be determined. Although the caudal spinal cord segments that control axial movements in tadpoles disappear with tail resorption after metamorphic climax, the segments above the lumbar enlargement are preserved in adulthood and must also adapt to the new body format. To what extent do developmental changes in the organization of segmental networks relate to the animal's needs for postural control during swimming? More specifically, what coordinating processes enable certain axial muscles, which are directly engaged in body propulsion 
in the pre-metamorphic larvae, to assume a dynamic postural function after their transformation into non-locomotory back muscles in post-metamorphic adults?

To begin to address these questions, we have analyzed the bilateral coordination of axial/back muscle activity in freely-behaving, pre-metamorphic Xenopus tadpoles and, together with the locomotor activity of hindlimb muscles, in post-metamorphic young adults. In the latter, moreover, simultaneous electromyographic (EMG) recordings were made from back and limb muscles on both sides of the body during free straight-ahead swimming, then a series of spinal cord lesions was performed to better understand the neural origins of the temporal relationships between activity in these muscle sets in vivo. We show that the rostro-caudal recruitment sequence and left-right alternation of thoracic axial muscles in the tadpole is replaced during metamorphosis by a different adult coordination pattern in which back and leg muscles contract synchronously during swimming, in a bilaterally in-phase pattern. Based on in vivo lesions and in vitro experiments on isolated brainstem-spinal cord preparations, it appears that the lumbar CPG for hindlimb locomotion is also directly responsible for driving thoracic motor output to dorsal muscles in post-metamorphic Xenopus. Given the developmental switch in function of the more rostral axial muscles in larvae to non-propulsive trunk musculature in the adult frog, the Xenopus model should help to provide new insights into the dynamic interactions between locomotory and postural control systems in general. Part of this work has been presented previously in abstract form (Beyeler et al. 2007). 


\section{MATERIALS AND METHODS}

Experiments were performed on four pre- and three pro-metamorphic tadpoles (stages 54 to 57 and to 60-61, respectively; Nieuwkoop and Faber 1956) and 50 postmetamorphic juvenile adults (stage 66, <1 month after metamorphosis) of the South African clawed toad, Xenopus laevis, bred from an in-house laboratory colony. Tadpoles were raised until near stage 50 at $20^{\circ} \mathrm{C}$ and then kept at room temperature throughout metamorphosis. EMG recordings were performed on animals at room temperature in separate aquaria, while in vitro experiments were performed at $16-18^{\circ} \mathrm{C}$. All procedures were in keeping with the animal care guidelines of the Bordeaux University and the CNRS.

In vivo electromyographic recordings

EMG activity of rostral axial musculature in larvae and back and leg muscles in juveniles was recorded using pairs of $50 \mu \mathrm{m}$ insulated wire electrodes connected through a grounded cable to a differential AC amplifier (Model 1700, AM-System Inc., WA). Axial muscles were recorded at the level of the $5^{\text {th }}$ segment of the tadpole spinal cord, corresponding to the segment that commands the future back muscles in postmetamorphic juveniles. In the latter, EMG recordings were made on both sides of the trunk from the third myomere of the dorsalis trunci, which is located dorsally along the vertebral column at mid-distance between the fore- and hindlimbs (Vallois 1922), and from the ankle extensor plantaris longus (often called gastrocnemius in frogs; Peters 2005) of both hindlimbs. The implantation of EMG recording wires was performed under light anesthesia after a small incision had been made in the overlying skin, then the electrodes were fixed to the muscle surface with a spot of Vetbond $3 \mathrm{M}$ adhesive 
(World Precision Instruments, UK). The skin was then replaced and attached with glue. EMG signals were directed to a computer through a CED Micro 1401 interface (Cambridge Electronic Design Ltd, UK) for storage and later analysis using Spike 2 (CED) software.

Surgery for spinal lesions in juveniles

Juvenile frogs were individually anesthetized with freshly dissolved tricaine methanesulfonate (MS 222, 50mg/L, Sigma-Aldrich) and positioned dorsal side up in a Petri dish filled with frog saline (in $\mathrm{mM}$ : $\mathrm{NaCl} 112 ; \mathrm{KCl} 2 ; \mathrm{NaHCO}_{3} 20 ; \mathrm{CaCl}_{2} 2,8$; $\mathrm{MgCl}_{2}$ 1; Glucose 17). After an incision was made in the dorsal skin, muscles were carefully removed and the underlying vertebrae were opened dorsally along the thoracic and/or lumbar cord regions. In order to separate the thoracic spinal cord from other regions of the nervous system, three types of lesion were made separately or in combination:

(i) a transection at the level of the last cervical cord segment;

(ii) a longitudinal (sagittal) lesion that extended through only the three thoracic segments;

(iii) a hemi- or whole-cord transection between the last thoracic and first lumbar segments;

After lesioning, the spinal cord was cleaned and covered with a small piece of gauze compress soaked in saline. The skin was then replaced and attached with Vetbond glue. Animals were allowed $24 \mathrm{hr}$ to recover from anesthesia and surgery before EMG recordings commenced. 
The extent of spinal lesions was verified after experimental recordings, and only data from animals with appropriate lesions were analyzed in this study. Control experiments on operated juvenile Xenopus but with the spinal cord remaining intact $(n=4)$ showed no significant changes in either their patterns of free swimming or the coordination between dorsalis trunci and plantaris longus muscle activity (data not shown). EMG recordings in pre- and pro-metamorphic tadpoles were restricted to rostral axial muscles at a level that corresponded to the adult thoracic cord region.

\section{Retrograde staining of dorsalis motoneurons}

The somata of motoneurons innervating the dorsalis trunci muscles were located in the spinal cord by means of retrograde axonal staining from target muscles in vivo. Four post-metamorphic animals were anesthetized in MS 222 and placed on ice in a Petri dish. A short incision was made in the skin along the dorsal midline in order to gain access to the back muscles. Small crystals of two fluorescent dyes (alexa fluor 488 and 546 coupled to dextran 10,000) were inserted separately with a fine pin into the left and right third myomeres of dorsalis trunci. The skin was then resealed with glue, and animals were placed in separate aquaria for recovery. After three days to allow retrograde dye migration across the neuromuscular junction and along motoneuron axons to the CNS, animals were sacrificed and their spinal cords dissected out and fixed overnight at $4{ }^{\circ} \mathrm{C}$ in $4 \%$ paraformaldehyde in phosphate buffer $0.1 \mathrm{M}, \mathrm{pH}=7.4$. After dehydration, the cords were cleared in glycerol and examined with a fluorescent microscope (Leica-DMRB, France) equipped with a CCD camera (ORCA-AG Hamamatsu, France). Images were acquired using Simple PCI software (Compix Inc., PA, USA). 


\section{Isolated brainstem-spinal cord preparations}

Dissection and recording procedures were similar to those previously described by Combes et al. (2004). The skin and cranial cartilage of juvenile frogs were opened under deep anesthesia with tricaine methanesulfonate and the forebrain removed above the mesencephalon. The spinal cord and remaining brainstem were dissected out together with the thoracic ventral roots and the nerve branches that innervate the hindlimb tibialis anterioris and the plantaris longus, which were previously identified as being ankle flexor and extensor muscles, respectively (d'Avella et al. 2003; d'Avella \& Bizzi, 2005). Isolated preparations were then transferred into a recording chamber and kept under oxygenated frog saline at $16-18^{\circ} \mathrm{C}$. Suction electrodes were used to record motor activity from left and right thoracic ventral roots, while Vaseline-insulated wire electrodes were used to record bilateral extensor and flexor activity "en passant" in distal limb motor nerve branches. Ventral root and nerve signals were amplified, displayed and stored in the same way as for EMG recordings.

All spinal motor output patterns, including those related to swimming, occurred spontaneously without additional chemical or electrical stimulation. Unfortunately, in vitro preparations subjected to cervico-thoracic or thoraco-lumbar transections were found incapable of producing bouts of coordinated fictive locomotion, either spontaneously or under classical pharmacological stimulation (with bath-applied NMDA, bicuculline, serotonin, noradrenalin, either separately or together). However, as with the intact spinal cord, isolated preparations with a sagittally-lesioned thoracic cord continued to generate episodes of spontaneous swimming motor output. Only data from these latter experiments were analyzed and are illustrated here. 


\section{Data analysis}

All analyses of electrical recordings were performed using homemade scripts running under Spike 2 (Spike 2 language, CED). Raw signals were first integrated, and only activity sequences obtained during episodes of rectilinear forward swimming were analyzed further. The onsets of motor bursts occurring during such episodes were automatically detected in the integrated traces, and pooled data according to animal groups (control, longitudinal spinal cord lesion, etc.) were then transferred to Oriana software (Kovach Computing Services, UK) for circular phase analysis of the temporal relationship between activity in selected pairs of muscles or nerves. The results of this analysis gave the mean vector $\mu$ and its length $r$, and two tests were used to examine the circular distributions: (i) the Rayleigh test $(Z)$ which tested the concentration of phase values around the mean vector, with a random distribution indicating a lack of coordination between the two compared burst activities and (ii), the V-test $(u)$ which tested for a preferential direction (angle) of a given phase distribution (indicated in the text as $\left.u\left(\operatorname{direction}^{\circ}\right)\right)$. In order to simplify graphical representations, phase values were plotted as grand means of the means of relative burst onsets throughout individual locomotor episodes. Nevertheless, the similarity between distributions (for example between control and lesioned animal groups) was verified for whole populations of events using the Mardia-Watson-Wheeler test $(W)$. The angular dispersion around the mean vector $\mu$ was also calculated in order to assess the effect of a given lesion on the power of coupling between different muscle or nerve discharges, with the size of the angular dispersion value being inversely proportional to the strength of coupling. A 
two-tailed non-parametric t-test was used to compare phase dispersions in groups of control and lesioned animals.

\section{$2 D$ kinematics in juvenile animals}

Intact and lesioned juvenile frogs were video-taped with a digital camera (Handycam DCR-PC350E, Sony, France) while behaving freely in their standard aquaria, or during EMG recording in a smaller experimental tank. No clear evidence was found for a perturbing effect of the fine EMG wires on the freedom of locomotor movements. The video files were transferred to a computer using Windows Movie Maker software (Microsoft, USA) for storage and joint kinematics. The latter were performed using the free Image $J$ software developed by W.S. Rasband (US National Institute of Health, MA, http://rsb.info.nih.gov/ij/, 1997-2005). Using a manual tracking plug-in supplied by F.P. Cordelières (Institut Curie, France), the $x$ and $y$ coordinates of limb joints were determined under visual inspection by mouse-clicking on individual frames. Joint angles were determined with Microsoft Excel, while stick diagrams and angle variations were plotted using CorelDraw 7 (Corel Corp., France). 


\section{RESULTS}

Developmental changes in bilateral axial muscle coordination during swimming in metamorphosing Xenopus

In pre-metamorphic Xenopus laevis (stages 54-57), forward locomotion is achieved by rhythmic body undulations produced by bilaterally-alternating spinal ventral root bursts that drive myotomal muscle contractions in rostro-caudally directed waves along the tail (Roberts et al. 1998; Combes et al. 2004). To observe such axial muscle activity in freely-behaving tadpoles, we recorded from the dorsal region of the myotome of the fifth spinal cord segment, which in the adult corresponds to the second thoracic segment controlling the back musculature. Consistent with earlier observations from spinal ventral root recordings in vitro (Combes et al. 2004), EMG recordings from left and right rostral axial muscles (hereafter referred to as axial/dorsal $(a d)$ ) in intact tadpoles were activated alternately during episodes of actual locomotion (Fig. 1A). The strongly clustered distribution of left versus right muscle burst onsets ( $n=7$ episodes; $\mu=170.00^{\circ}$; $r=0.99$; Fig. 1 A right) was further indicative of a strong phase-coupling $(Z=6.84$; $\mathrm{p}<0.001)$ with a phase difference of near $180^{\circ}\left(u\left(180^{\circ}\right)=3.64 ; \mathrm{p}<0.001\right)$ corresponding to symmetrical left-right alternating contractions necessary for rectilinear propulsion.

During metamorphosis, anurans like Xenopus develop articulated limbs that become functional while the larval tail continues to play an active locomotor role for 1 to 2 weeks prior to its degeneration (Nieuwkoop and Faber 1956; Combes et al. 2004). During this transitional period, both axial and appendicular locomotor systems can participate conjointly or independently in swimming behavior. As seen in the EMG recordings from a pro-metamorphic (stage 60-61) tadpole in Figure 1B, the axial/dorsal muscles display two distinct types of bilateral coordination according to the mode of 
locomotion. During tail-based swimming ( $\mathrm{n}=9$ episodes; Fig. 1B1), the left and right $a d$ muscles express a typical larval coordination pattern in which they are activated alternately $\left(\mu=152.11^{\circ} ; r=0.93 ; Z=6.04 ; \mathrm{p}<0.001 ; u\left(180^{\circ}\right)=3.07 ; \mathrm{p}<0.001\right)$, whereas following a spontaneous switch to limb-based propulsion (12 episodes; Fig. 1B2), the same $a d$ muscles are now active in synchronous bursts $\left(\mu=350.53^{\circ} ; r=0.98 ; Z=9.63\right.$; $\left.\mathrm{p}<0.001 ; u\left(0^{\circ}\right)=4.33 ; \mathrm{p}<0.001\right)$.

Following metamorphosis (stage 66;17 animals) the more rostral axial muscles, which now correspond to postural back muscles (dorsalis trunci, referred to as " $d t$ " in Fig. 1C and following figures), continue to be co-activated during swimming that is now solely limb-based following resorption of the tail. Thus, although uncoordinated bursts may also occur occasionally (not illustrated), circular analysis of left versus right dorsalis trunci bursts revealed a relatively narrow phase distribution $(\mathrm{n}=37$ episodes; $\left.\mu=358.59^{\circ} ; r=0.98 ; Z=35.39 ; \mathrm{p}<0.001\right)$ that had a preferential direction toward phase $0^{\circ}$ $\left(u\left(0^{\circ}\right)=8.41 ; \mathrm{p}<0.001\right)$. These findings therefore show that during metamorphosis, dorsal axial muscles alter their pattern of activation during swimming from strict bilateral alternation during tail-based locomotion to predominant synchrony during limb-based propulsion. Moreover, in mixed metamorphic stages when both tail- and limb-based propulsion occurs, the $a d$ muscles of a given animal can switch, even within the same swimming episode, between either coordination pattern according to the type of locomotion being expressed. While the neural basis of axial muscle recruitment during swimming in pre-metamorphic Xenopus tadpoles has been well described (Roberts et al. 1998), the coordinating mechanisms engaged in driving these muscles during limb-based locomotion in the adult are unknown. The following in vivo and in vitro experiments were therefore designed to explore the neural origin of the 
synchronous activation of bilateral dorsal trunk muscles during forward swimming in post-metamorphic animals.

Coordination of hindlimb and dorsal muscles during rectilinear swimming in juvenile adults

The hindlimb muscle activity patterns that allowed a distinction to be made between rectilinear forward swimming and turning behavior can be seen in the kinematics analysis and associated EMG recordings of Figure 2. Episodes of linear swimming are characterized by bilaterally-symmetrical kick trajectories of the hind legs (see stick diagrams in Fig. 2A1), unlike the uncoordinated limb movements associated with nonlinear swimming (Fig. 2B1). As can be seen in the traces of the hindlimb knee and ankle joint excursions in Figure 2A2, B2 (upper traces), the coordinate movements of the two right limb joints occurred as an in-phase mirror image of the homologous left limb joint excursions during a linear swim episode (Fig. 2A2). In contrast, the excursions of these same joints became asymmetric and irregular during turning maneuvers (Fig. 2B2). Correspondingly, in simultaneous left-right EMG recordings, the ankle extensor plantaris longus muscles (referred to as " $p l$ " in Fig. 2 and following figures) of the hindlimbs were synchronously active with regularly-recurring bursts during rectilinear swim episodes (Fig. 2A2, lower traces; see also Fig. 3B,C), whereas the activity of the same muscles became desynchronized during non-linear swimming (Fig. $2 \mathrm{~B} 2$ bottom traces). In the remaining in vivo and in vitro experiments, therefore, we focused attention on episodes of real or fictive swimming in which synchronous bursting occurred either in the left and right plantaris muscles or in the corresponding extensor motor nerves. 
In order to determine how back and hindlimb motor activity is coordinated during young adult swimming, we made simultaneous EMG recordings in vivo from the left and right plantaris longus muscles and the third myomeres of dorsalis trunci (Fig. 3A, B). An initial analysis of the phase relationship between left and right plantaris longus burst onsets showed a close coordination ( $\mathrm{n}=21$ episodes; $\mu=4.89^{\circ} ; r=0.98 ; Z=20.04$; $\mathrm{p}<0.001$; Fig. $3 \mathrm{C}$ middle polar plot) that was centered around a $0^{\circ}$ phase value $\left(u\left(0^{\circ}\right)=6.31 ; \mathrm{p}<0.001\right)$. Thus, although some phase dispersion occurred, the main tendency was for bilateral synchrony to occur between the two extensor muscles, commensurate with the generation of linear forward propulsion (see Fig. 2A, compare with 2B).

In addition to the bilateral synchrony of dorsalis trunci muscle activity during swimming (Fig. 1C and Fig. 3B,C left polar plot), a phase analysis of dorsalis EMG activity versus ipsilateral plantaris bursts ( $\mathrm{n}=23$ episodes; $\mu=3.59^{\circ} ; r=0.96 ; Z=21.37$; $\mathrm{p}<0.001$; Fig. $3 \mathrm{C}$ right polar plot) also indicated coincident discharge of these trunk and limb muscles (also see Fig. 3B), with a mean burst onset phase vector that was directed at $0^{\circ}\left(u\left(0^{\circ}\right)=6.53 ; \mathrm{p}<0.001\right)$. Thus, in contrast to the rostro-caudal propagation of alternating contractions of the equivalent muscles in the pre-metamorphic tadpole, the relative timing of dorsal muscle contractions had altered with the change in mode of locomotion during metamorphosis, so that the activation of dorsalis trunci in the froglet was now phase-locked with bilaterally-synchronous hindlimb movements.

Furthermore, the strength of activation of the dorsal and hindlimb extensor muscle pairs displayed similar relationships with swimming speed (mean frequency $4.4 \pm 1.2 \mathrm{~Hz}$; 17 animals). In both cases, area measurements of individual bursts in the integrated EMG traces revealed a linear and slightly positive correlation between burst area and 
the frequency of the corresponding swim cycle (regression coefficient $\left(r_{c}\right)=0.44$, $\mathrm{p}<0.001$, regression slope $(a)=0.11$ and $r_{c}=0.54, \mathrm{p}<0.001, a=0.09$ for dorsalis and plantaris muscles, respectively; $n=450$ cycles). Moreover, the intensities of back versus limb muscle bursts were themselves significantly and positively correlated $\left(r_{c}=0.24\right.$, $\mathrm{p}<0.001, a=0.37$ ), which further pointed to the close temporal relationship between locomotor-related output produced in the thoracic and lumbar cord regions.

We next investigated whether this coupling between dorsalis trunci and plantaris longus activity was mediated by direct propriospinal interactions between the thoracic and lumbar cord regions or resulted indirectly from sensory reflexes, as has been reported to occur, for example, in the maintenance of human stance (Tokizane et al. 1951). To this end, an in vitro brainstem/spinal cord preparation, therefore with no movement-related sensory feedback, was used to assess the effects of deafferentation on the temporal relationships between motor activity recorded in the second thoracic ventral roots that normally innervate dorsalis trunci and the distal nerve branches innervating the ankle extensor and flexor muscles (Fig. 3D). As previously reported (Combes et al. 2004), such isolated preparations $(n=7)$ continue to express spontaneous episodes of fictive locomotion (Fig. 3E). Although generally shorter (8.2 \pm 3.5 vs. $10.5 \pm 2.9$ cycles; $\mathrm{p}<0.05)$ and slower $(1.77 \pm 0.5$ vs. $4.4 \pm 1.2 \mathrm{~Hz} ; \mathrm{p}<0.001)$ than swimming episodes in the intact animal, these in vitro motor patterns continued to exhibit burst phase relationships that corresponded closely to trunk and limb movements in vivo (Fig. 3F). Thus, homolateral antagonistic limb nerves were active in alternation $\left(\mu=186.06^{\circ} ; r=0.64 ; Z=174.04 ; p<0.001\right)$, while homologous bilateral motor nerves tended to express in-phase bursting (tibialis nerves: $\mu=26.59^{\circ} ; r=0.69 ; Z=134.55$; $\mathrm{p}<0.001$ : plantaris nerves: $\mu=343.12^{\circ} ; \quad r=0.84 ; Z=190.99 ; \mathrm{p}<0.001$ ), in a manner 
appropriate for producing alternating cycles of bilaterally-synchronous limb extensions and flexions. In addition, burst synchrony was preserved across the isolated cord at the thoracic segmental level (Fig. 3E,F: $\mu=2.58^{\circ} ; r=0.53 ; Z=134.55 ; \mathrm{p}<0.001$ ) as well as between the thoracic ventral roots and plantaris nerves originating from the lumbar cord region $\left(\mu=2.22^{\circ} ; r=0.63 ; Z=97.97 ; \mathrm{p}<0.001\right)$. These results therefore clearly imply that sensory inputs generated by actual limb and body movements during locomotion are not essential for maintaining either the coordination of thoracic motor output to the left and right back muscles or the longitudinal coupling between motor commands to the back and hindlimb muscles. Nonetheless, a significant increase in the phase dispersion of bilateral thoracic bursts in the isolated preparation $(\mathrm{p}<0.05$; Fig. $3 \mathrm{G}$, compare left pair of histogram bars) indicated that sensory information may participate in strengthening the coordination between the left and right dorsalis muscle contractions. However, other burst phase relationships (left vs. right plantaris longus and dorsalis vs. plantaris) remained similarly distributed in vitro to their corresponding muscle EMG patterns in vivo (Fig. 3G, middle and right histogram pairs).

Effects of spinal lesions on the coordination between juvenile hindlimb and dorsal muscle activity

To further understand the phase-coupling of back and hindlimb muscle contractions during linear forward swimming, we made specific lesions to the spinal cords of juvenile frogs (see Methods) in order to define the structural substrate necessary for ensuring such a strong functional relationship. 


\section{$\underline{\text { Cervico-thoracic cord lesions }}$}

In higher vertebrates including humans, it is commonly acknowledged that the coordination between postural back muscle activity and limb locomotor movements is largely governed by descending influences from supra-spinal centers (Takakusaki et al. 2004; Lalonde and Strazielle 2007). To assess whether, in the lower vertebrate Xenopus, dorsalis trunci activation and its coordination with hindlimb movements might also depend on cerebrospinal commands, a series of in vivo and in vitro experiments was performed in which a complete spinal cord transection was made between the last cervical and the first thoracic segments. Unfortunately, no consistent patterns of fictive swimming could be evoked in isolated preparations after such a cervico-thoracic cord transection ( $\mathrm{n}=8$; not shown), and only lesions performed in vivo provided useful data $(n=11$; Fig. 4). Although these lesioned animals were unable to produce spontaneous locomotor-related activity, tactile stimulation of one of the hindlimbs could immediately elicit short episodes of linear swimming kick movements that were similar to those expressed in intact animals (Fig. 4A). Limb and trunk kinematics during such episodes were also comparable to those of intact animals (not shown), and again, the left and right plantaris longus muscles remained active in strict synchrony ( $\mathrm{n}=7$ episodes; $\mu=2.84^{\circ} ; r=0.99 ; Z=6.86 ; \mathrm{p}<0.001 ; u\left(0^{\circ}\right)=3.71 ; \mathrm{p}<0.001 ;$ Fig. $4 \mathrm{~B}$, middle polar plot). However, the coupling between these muscles was increased significantly by the rostral cord transection $(W=24.68 ; \mathrm{p}<0.001)$ as seen in the middle histogram pair of Figure $4 \mathrm{C}$, where the phase dispersion in lesioned animals (filled histogram bars) was compared with equivalent data obtained from intact control animals (as illustrated in Fig. 3A-C, G). In cervico-thoracic transected animals, the activity of left and right dorsalis trunci also remained synchronized $\left(\mathrm{n}=26\right.$ episodes; $\mu=5.87^{\circ} ; r=0.97 ; Z=24.44 ; \mathrm{p}<0.001$; 
$u\left(0^{\circ}\right)=6.95 ; \mathrm{p}<0.001$; Fig. 4B left $)$, with the phase dispersion also being significantly narrower $(W=34.21 ; \mathrm{p}<0.001)$ than in intact animals (Fig. 4C, left histogram pair). Similarly, back and leg muscle activity continued to display an in-phase coordination $\left(\mathrm{n}=7\right.$ episodes; $\mu=2.20^{\circ} ; r=0.98 ; Z=5.74 ; \mathrm{p}<0.001 ; u\left(0^{\circ}\right)=3.39 ; \mathrm{p}<0.001 ;$ Fig. 4B right $)$ with a burst onset phase distribution significantly different from control ( $W=17.38$; $\mathrm{p}<0.001$ ) due to a significant decrease in phase dispersion (Fig. $4 \mathrm{C}$ right). As in control animals, furthermore, the intensity of dorsal and limb extension muscle bursts remained linearly correlated $\left(r_{c}=0.72 ; \mathrm{p}<0.001 ; a=0.82\right)$ after a cervico-thoracic transection. These data therefore indicated that the cyclic activation of dorsalis trunci muscles during locomotion was not attributable to descending commands from the brainstem. Rather, the actual strengthening of thoracic cross-cord and lumbo-thoracic coupling following a rostral cord transection was more indicative of the functional versatility that brainstem influences are capable of imposing on otherwise inflexible coordination patterns arising from hardwired spinal circuitry.

\section{$\underline{\text { Sagittal lesions to the thoracic spinal cord }}$}

In pre-metamorphic Xenopus embryos, the left-right alternation of axial muscle contractions derives from intra-segmental inhibitory connections between locomotory circuitry on the two sides of the cord (Soffe and Roberts 1982; for a review see Roberts et al. 1998). In order to assess whether the switch to bilateral synchrony of dorsalis muscle activation in the adult animal might result from the establishment of cross-cord excitatory connections during metamorphosis, restricted midline cord sagittal lesion were made in froglets to separate the two sides of all three segments of the thoracic cord region. After such a sagittal lesion (n=7; Fig. 5A-D) animals were still able to express 
rectilinear swimming-like behavior (Fig. 5B), and EMG recordings as well as kinematics (not illustrated) did not reveal any major alterations in the pattern of coordination either between bilateral hindlimb movements or between the contractions of the left and right dorsalis trunci muscles. Thus, the two plantaris longus muscles were still synchronously active during swimming episodes $\left(\mu=4.28^{\circ} ; r=0.80 ; Z=178.57\right.$; $\mathrm{p}<0.001 ; u\left(0^{\circ}\right)=18.84 ; \mathrm{p}<0.001 ;$ Fig. $\left.5 \mathrm{C}\right)$ with a coupling strength that remained similar to intact control animals ( $W=2.93 ; \mathrm{p}>0.05$; and Fig. 5D middle histogram pair). In contrast, although the left and right dorsalis trunci also retained an overall tendency for synchronous activity $\left(\mu=14.85^{\circ} ; \quad r=0.54 ; Z=72.79 ; \mathrm{p}<0.001 ; u\left(0^{\circ}\right)=11.66 ; \mathrm{p}<0.001 ;\right.$ Fig. 5C, left polar plot), the burst onset phases were slightly but significantly more dispersed than in control ( $\mathrm{W}=35.52 ; \mathrm{p}<0.001$; Fig. 5D, left histogram pair). However, a closer inspection of the raw data revealed that this broader distribution resulted from an increase in the occurrence of supernumerary dorsalis activity that did not correspond to hindlimb muscle contractions (e.g. L $d t$ EMG activity in Fig. 5B). Nevertheless, these data indicated that excitatory cross-cord pathways within the thoracic segments were not primarily responsible for the bilateral synchronization of dorsal muscle activity (see below).

Significantly, a thoracic cord partitioning did not alter the linear positive relationship between the relative strengths of dorsalis and plantaris activation $\left(r_{c}=0.56\right.$; $\mathrm{p}<0.001, a=0.37)$, and their burst firing became more tightly coupled $\left(\mu=1.88^{\circ} ; r=0.82\right.$; $Z=160.23 ; \mathrm{p}<0.001 ; u\left(0^{\circ}\right)=17.90 ; \mathrm{p}<0.001 ;$ Fig. $5 \mathrm{C}$ right polar plot $)$ than in control animals $(W=17.21 ; \mathrm{p}<0.001)$, as indicated by the reduction in phase dispersion seen in Figure 5D (right histogram pair). This stronger coordination between plantaris and dorsalis activity after a thoracic sagittal lesion was further confirmed in three additional 
in vivo experiments, where a thoracic midline section was made in conjunction with both a cervico-thoracic transection and a dorsal rhizotomy of the thoracic and lumbar cord segments. The overall pattern of lumbo-thoracic activation not only persisted after such multiple lesions, but they also significantly enhanced the coupling between left vs. right plantaris longus and dorsalis vs. plantaris muscle activity, as indicated by the substantial increase in $r$ values for all phase relationships presented in Table 1 . These latter experiments therefore supported the conclusion that propriospinal projections from the lumbar CPG circuitry to thoracic cord segments are sufficient and necessary for maintaining the strict lumbo-thoracic coupling observed during normal rectilinear swimming.

Finally, sagittal thoracic cord lesions to in vitro preparations (Fig. 5E) had effects on fictive locomotor patterns $(n=4$; Fig. $5 \mathrm{~F}-\mathrm{H})$ that were broadly similar to those seen in vivo (compare with Fig. 5A-D). This included a persistence of overall synchrony between left and right thoracic ventral root bursts $(W=5.75 ; \mathrm{p}>0.05 ;$ Fig. $5 \mathrm{G}$ left $)$ despite a significantly increased phase dispersion ( $<<0.05$; Fig. $5 \mathrm{H}$ left), which in turn, and consistent with in vivo lesion experiments (Fig. 5D left), implied a residual contribution of thoracic cross-cord connections to bilateral coupling. More significantly, no changes were observed in the overall coordination between locomotor-related bursting in the plantaris longus motor nerves and thoracic ventral roots after a thoracic sagittal section in vitro (Fig. 5F, compare with recordings from intact isolated cord in Fig. 3E), although inexplicably, the strength of the coupling between both left and right limb extensor and flexor nerve bursts increased significantly (bilateral tibialis nerves, $W=54.47 ; \mathrm{p}<0.001:$ plantaris nerves, $W=78.07 ; \mathrm{p}<0.001)$ due to a decreased variability in their phase distributions (Fig. $5 \mathrm{H} ; \mathrm{p}<0.01$ and $\mathrm{p}<0.001$, respectively). 


\section{$\underline{\text { Thoraco-lumbar cord lesions }}$}

In order to confirm that the phasic activation of the left and right dorsalis trunci derives directly from the lumbar locomotory $\mathrm{CPG}$, complete spinal cord transections were made between the last thoracic and first lumbar segments. Here again, such lesions prevented locomotor-related activity in in vitro preparations ( $\mathrm{n}=8$; not shown), and meaningful data was only obtained from in vivo experiments $(\mathrm{n}=11$; Fig. 6A-C) in which the dorsalis or plantaris muscles were activated by briefly pinching either an anterior or a posterior limb, respectively. With hindlimb stimulation (Fig. 6A1), only arrhythmic and mostly uncoordinated plantaris muscle activity was observed. In contrast, EMG-recorded dorsalis (i.e. at a level more rostral to the cord transection) expressed left and right patterns of activity (Fig. 6A2 and left plot in Fig. 6B) in response to a forelimb stimulation (which also elicited bouts of forelimb movements; not illustrated) that tended to be synchronous $\left(\mu=359.42^{\circ} ; r=0.73 ; Z=22.05 ; \mathrm{p}<0.001\right.$; $\left.u\left(0^{\circ}\right)=6.64 ; \mathrm{p}<0.001\right)$ despite a wider phase dispersion $(W=12.08 ; \mathrm{p}<0.01 ;$ and Fig. $6 \mathrm{C}$ left) than in intact control animals. As expected, no temporal correlation was found between dorsalis activity and the occasional plantaris bursts that occurred in response to hindlimb pinching $\left(\mu=302.16^{\circ} ; r=0.69\right.$; but $Z=1.40 ; p>0.05$; Fig. $6 \mathrm{~B}$ right), further confirming that dorsalis was not being driven via a possible reflex loop that was being activated by passive dorsal muscle stretching during hindlimb extensions.

Finally, in two juveniles, the thoracic and lumbar segments were separated on one side only (left) in combination with a longitudinal thoracic segment section. Such sagittally/hemi-cord lesioned animals were still able to swim spontaneously (Fig. 6D1) or in response to limb tactile stimulation (not illustrated). Under these conditions, 
dorsalis activity generally occurred only on the unlesioned side (Fig. 6D1) and it remained phase-coupled to rhythmic hindlimb extensions $\left(\mu=334.12^{\circ} ; r=0.92 ; Z=6.73\right.$; $\mathrm{p}<0.001 ; u\left(0^{\circ}\right)=3.30 ; \mathrm{p}<0.001 ;$ Fig. $6 \mathrm{E}$, right plot). In contrast, the occasional dorsalis activity that occurred on the side of the hemi-cord lesion expressed no clear temporal relationship with hindlimb plantaris contractions $\left(\mu=128.89^{\circ} ; r=0.73 ; Z=4.31 ; p<0.001\right.$; non significant V test; Fig. 6E, left plot). Consequently, the dorsalis vs. plantaris phase values for activity on the disconnected (left) cord side was significantly more dispersed than for the intact (right) cord side ( $<<0.001$; Fig. 6F). Finally, these bilateral differences were not the result of lesion-induced damage to ipsilateral thoracic motor circuitry since strong spontaneous bilateral dorsalis activity was occasionally observed when hindlimb locomotor movements were absent (Fig. 6D2). 


\section{DISCUSSION}

The fundamental modifications in locomotor behavior that occur during amphibian metamorphosis (Combes et al. 2004; Rauscent et al. 2007) require a concomitant adaptation of postural control and its coordination with locomotion. The present study explored this changing functional relationship by recording axial/thoracic motor output to locomotory muscles in pre-metamorphic Xenopus tadpoles and after their transition to postural musculature in post-metamorphic young adults. Associated changes in longitudinal coupling between thoracic and lumbar cord segments during swimming in juvenile frogs were also examined through simultaneous recordings from respective back and hindlimb muscles, from their corresponding motor nerves in isolated nervous system preparations, and after various spinal cord lesions performed either in vivo or in vitro.

\section{Metamorphosis-induced modifications in intra- and inter-segmental coordination}

Whereas in tadpoles the entire axial musculature is recruited in strict left/right alternation, the dorsal axial muscles controlled by the thoracic region of the young adult spinal cord are synchronously activated during free forward swimming. Moreover, in contrast to the progressive rostro-caudal activation of axial muscles in swimming larval Xenopus (Roberts et al. 1998; Combes et al. 2004), back and leg extensor muscles are co-activated during juvenile hindlimb propulsion. These radical changes in the coordination between left and right thoracic hemi-segments, and between thoracic and lumbar segments, therefore implied that the functional reorganization of spinal motor circuitry that occurs during Xenopus metamorphosis (Combes et al. 2004) extends into mid cord regions that are not involved in producing actual limb movements. Several 
observations from our in vivo and in vitro experiments provided insights into the nature of this developmental transformation.

Firstly, all spinal lesions in froglets that isolated the thoraco-lumbar cord from sensory or supra-spinal inputs tended to narrow the phase distributions of back versus leg muscle locomotor activity towards even closer synchrony, suggesting that a preferential functional linkage between the lumbar and thoracic cord segments is established during the metamorphic process. Further clear evidence that the dorsal muscle-hindlimb extensor coupling does not rely on sensory feedback arises from the observation that the activation patterns of thoracic ventral roots and leg motor nerves in isolated in vitro preparations remained similar to those of their corresponding target muscles in vivo. The stronger synchrony observed between back and hindlimb muscle activity after disconnecting the thoraco-lumbar cord from the rostral CNS also suggested that the coupling was not reliant upon descending influences from the brainstem, a situation that differs from rodents and humans where supra-spinal inputs have been reported to be primarily responsible for coordinating back and leg muscle contractions during locomotion (Gramsbergen et al. 1999; Takakusaki et al. 2004). Thus, although a remodeling of supra-spinal centers is undoubtedly required in metamorphosing Xenopus to adapt descending commands to the changing spinal circuitry, the altered thoraco-lumbar coordination occurring in young adults is mainly attributable to de novo synaptic pathways that are intrinsic to the spinal cord.

Secondly, that the left/right back muscle synchronization with bilateral hindlimb extensions persisted after separating the two sides of the thoracic cord suggested that the back muscle coupling does not rely principally on direct cross-cord connections at the thoracic cord level. This contrasts with the situation in pre-metamorphic Xenopus larvae 
where inhibitory commissural interneurons are responsible for alternating segmental CPG activity on the two sides of the cord (Roberts et al. 1998). Evidently, these larval inhibitory interactions are not simply replaced by equivalent excitatory cross-cord pathways in thoracic segments during metamorphosis in order to ensure conjoint left/right dorsalis activation. Rather, the persistence of dorsal muscle co-activation after thoracic cord partitioning which remained coordinated with hindlimb movements indicated that the thoracic intra-segmental coupling was being driven by the lumbar CPG itself. In direct contrast to the thoracic segments, however, the assembly of hindlimb locomotor circuitry in the lumbar cord region during metamorphosis must here include new cross-cord excitatory connections that are necessary for bilateral limb kick synchrony (see also Combes et al. 2004). Furthermore, the establishment of longitudinal projections from the lumbar to thoracic cord regions is also required to provide the necessary anatomical substrate for coupling dorsalis efferent commands to the hindlimb motor circuitry. While such ascending propriospinal pathways remain to be identified in post-metamorphic Xenopus, in Rana pipens, lumbar interneurons have been previously labeled which project directly into the thoracic spinal cord (Schotland and Tresch 1997). In Xenopus juveniles, furthermore, these lumbo-thoracic projections appear to be homolateral, since a hemicord section in vivo that disconnected the thoracic circuitry of that side from the lumbar cord region suppressed activity of the corresponding dorsalis muscle during swimming, but did not affect hindlimb kicking-timed activation of the dorsalis on the intact contralateral side.

Finally, a complete cord transection that separated the thoracic and lumbar segments not only decoupled leg and back motor outputs during swimming, but also resulted in an overall significant decrease in coincident left-right dorsalis activity. However, this 
bilateral relationship never became completely random after a thoraco-lumbar separation, indicating that co-activating influences on the motor commands to these muscles may to some extent arise from cerebrospinal inputs and/or the cervical CPG circuitry responsible for rhythmic forelimb movements. Indeed, it is possible that the occasional participation of forelimb CPG-driven propulsion during hindlimb swimming may also contribute to the coordination of dorsalis muscle contractions.

Taken together, therefore, these findings indicate that descending pathways, sensory inputs and cross-cord connectivity in the thoracic spinal cord are not essential for coordinating bilateral back muscle contractions with rhythmic hindlimb extensions during rectilinear swimming in juvenile Xenopus. Rather, their coordination appears to be determined principally by ipsilateral projections that ascend from the hindlimb CPG in lumbar cord segments. Earlier work on spinal cat has also proposed that the lumbar locomotor CPG may control the activation sequence of back muscles during walking (Zomlefer et al. 1984), and in newborn rats, changes in trunk curvature during locomotion are time-locked with rhythmic hindlimb stepping, which here also appears to derive from lumbo-thoracic interactions (Cazalets 2005; Falgairolle and Cazalets 2007).

It is important to remember that the development of limb-based locomotion in Xenopus is fundamentally different from most other vertebrates in which effective locomotory behavior depends on the progressive emergence of functional spinal circuitry from a non-functional embryonic precursor. In Xenopus, an already operational primary locomotor system (for axial-based swimming) is replaced by another during metamorphosis to satisfy completely different biomechanical requirements (for limbbased propulsion). Figure 7 summarizes the associated developmental changes in spinal 
network coordination on the basis of previous knowledge on larval Xenopus (Tunstall and Roberts 1994; Roberts et al. 1998) and our current findings in young adult frogs. Essentially, spinal connectivity switches from a system that generates rostro-caudally propagating, bilaterally-alternating motor patterns for undulatory swimming in tadpoles (Fig. 7A) to caudo-rostrally, bilaterally-synchronous activity that drives back muscle contractions in time with limb-kicking in the adult (Fig. 7B). In this way, thoracic circuitry, which contributes actively to body propulsion as an equivalent member of a chain of segmental oscillatory networks in the pre-metamorphic larva, becomes subordinate to new, more caudal, rhythm-generating circuitry that drives hindlimb extension/flexion from the lumbar region of the adult spinal cord.

\section{Posturo-locomotor interactions}

For effective displacement during locomotion, coordinated dynamic postural regulation is required to stabilize body orientation by controlling mainly the head and trunk musculature (Massion et al. 2004; Assaiante et al. 2005). The interactions between locomotory and postural systems are generally considered to be based on two separate neural command networks, both of which are thought to be activated and coordinated exclusively by supra-spinal commands and sensory feedback signals (see Mori et al. 2004). Although the postural trunk muscles of mammals, like in adult Xenopus, are known to contract rhythmically in time with the step cycle (Koehler et al. 1984; Gramsbergen et al. 1999; Falgairolle and Cazalets 2007), the neural basis of this coupling has remained unclear, due undoubtedly to the close functional relationship between the locomotory and postural systems that renders them difficult to distinguish during ongoing movement. Furthermore, in most species including humans, the two 
systems develop simultaneously and often employ overlapping sets of muscles (Vinay et al. 2002).

In lower vertebrates such as fish, non-anuran amphibians and anuran larvae (including Xenopus), locomotion and posture are achieved by the same sets of axial muscles that are activated sequentially along the body length during swimming, indicating that the coordination between these two functions occurs at the individual segmental level (Deliagina et al. 2006). In post-metamorphic anurans where propulsion is now limb-based, at least part of the larval dorsal axial musculature has evidently undergone a functional switch from a mainly locomotory to an essentially postural role as thoracic trunk muscles in the adult. Specifically, given its location flanking a rigidified section of the adult spinal cord, and consistent with a requirement for dynamic postural control in general (Massion 1994), the most likely contribution of the third myomere of dorsalis trunci during locomotion, especially during powerful swimming, is to increase body stiffness in order to resist external forces, in a similar manner to epaxial musculature in lizards, for example, which serves to stabilize the trunk against ground reaction forces during locomotion (Ritter 1995, 1996).

In lower vertebrates and mammals, both anticipatory and compensatory adjustments to postural perturbations are thought to depend on supra-spinal influences and sensory feedback from the periphery (Mori 1987; Mori et al. 2004; Deliagina et al. 2006), thereby implying that spinal postural circuitry serves merely as a final output stage for higher order or reflex commands. Our findings add a further dimension to this common view by indicating that the spinal cord is itself capable of organizing a major component of postural adjustment by using intrinsic feed-forward signals from the lumbar patterngenerating networks to predict the consequences of locomotor actions. Interestingly, 
previous work on cats has shown that vestibular information is not required for controlling posture, since responses to postural perturbations are preserved following a bilateral labyrinthectomy (Macpherson and Inglis 1993). Furthermore, complex motor kinematics and EMG coordination (Bélanger et al. 1996), and body orientation and geometry (Fung and Macpherson 1999) persist in chronic spinal cats, indicating here also that supra-spinal commands are not essential for maintaining equilibrium during walking or quiet stance. However, to our knowledge a proactive (rather than an anticipatory or reactive) relationship between locomotory movements and postural adjustments that resides solely with propriospinal mechanisms has not previously been clearly established (Stuart 2005).

In conclusion, Xenopus metamorphosis is associated with a complete reorganization of the postural system from a larval architecture in which the rostro-caudal recruitment of axial musculature during swimming implies postural adjustments on a segment-bysegment basis, to an adult "en bloc" strategy of postural control where posturolocomotor coordination extends over several spinal segments, from the lumbar to the thoracic cord regions. Furthermore, our findings suggest that Xenopus offers an attractive model for future studies on the developmental plasticity of posture/locomotion coupling and spinal network interactions. Especially intriguing in this context is that both larval and adult motor networks co-exist and can function separately within the spinal cord at mid-metamorphic stages of development (see Fig. 1B), thereby providing the opportunity to explore the neuronal basis of different posturo-locomotory strategies within the same organism. 


\section{Acknowledgements and grants}

The authors are grateful to M. Falgairolle for helping with kinematics analysis, and R. Nargeot for advice in statistical treatments. We also thank M.-J. Cabirol-Pol for technical assistance in fluorescence microscopy and L. Parra-Iglesias for animal care and maintenance. This work was funded by the CNRS (ATIP Jeunes Chercheurs). A. Beyeler was funded by a studentship from the French "Ministère de L'Education Nationale, de l'Enseignement Supérieur et de la Recherche". The authors also thank the European Neuroscience Institute Network (ENI-Net) for valuable discussions and for covering part of the publication fees. 


\section{REFERENCES}

Assaiante C, Malau S, Viel S, Jover M, Schmitz C. Development of postural control in healthy children: a functional approach. Neural Plast 12: 109-118, 2005.

d'Avella A, Bizzi E. Shared and specific muscle synergies in natural motor behaviors. Proc Natl Acad Sci USA 102: 3076-3081, 2005.

d'Avella A, Saltiel P, Bizzi E. Combinations of muscle synergies in the construction of a natural motor behavior. Nat Neurosci 6: 300-308, 2003.

Ballion B, Morin D, Viala D. Forelimb locomotor generators and quadrupedal locomotion in the neonatal rat. Eur J Neurosci 14: 1727-1738, 2001.

Bélanger M, Drew T, Provencher J, Rossignol S. A comparison of treadmill locomotion in adult cats before and after spinal transection. J Neurophysiol 76: 471491, 1996.

Beyeler A, Métais C, Combes D, Simmers J, Le Ray D. Propriospinal coordination of posturo-locomotor function in post-metamorphic Xenopus laevis. Program No. 187.3 Neuroscience Meeting Planner. San Diego, CA: Society for Neuroscience. Online, 2007.

Cazalets JR. Metachronal propagation of motoneurone burst activation in isolated spinal cord of newborn rat. J Physiol 568:583-597, 2005.

Cazalets JR, Bertrand S. Ubiquity of motor networks in the spinal cord of vertebrates. Brain Res Bull 53: 627-634, 2000.

Cazalets JR, Borde M, Clarac F. Localization and organization of the central pattern generator for hindlimb locomotion in newborn rat. J Neurosci 15: 4943-4951, 1995.

Combes D, Merrywest SD, Simmers J, Sillar KT. Developmental segregation of spinal networks driving axial- and hindlimb-based locomotion in metamorphosing Xenopus laevis. J Physiol 559: 17-24, 2004.

Deliagina TG, Orlovsky GN, Zelenin PV, Beloozerova IN. Neural bases of postural control. Physiology 21: 216-225, 2006.

Falgairolle M, Cazalets JR. Metachronal coupling between spinal neuronal networks during locomotor activity in newborn rat. J Physiol 580: 87-102, 2007.

Fung J, Macpherson JM. Attributes of quiet stance in the chronic spinal cat. $J$ Neurophysiol 82: 3056-3065, 1999. 
Gramsbergen A, Geisler HC, Taekema H, van Eykern LA. The activation of back muscles during locomotion in the developing rat. Dev Brain Res 112: 217-228, 1999.

Grillner S. Control of locomotion in bipeds, tetrapods and fish. In : Handbook of Physiology. The Nervous system. Motor control. Vol. II, part 2. Ed : Brooks, V.; Am. Physiol. Soc., Bethesda (MD), 1981, pp. 1179 - 1236.

Grillner S. Biological pattern generation: the cellular and computational logic of networks in motion. Neuron 52: 751-766, 2006.

Kiehn O. Locomotor circuits in the mammalian spinal cord. Annu Rev Neurosci 29: 279-306, 2006.

Koehler WJ, Schomburg ED, Steffens H. Phasic modulation of trunk muscle efferents during fictive spinal locomotion in cats. J Physiol 353: 187-197, 1984.

Lalonde R, Strazielle C. Brain regions and genes affecting postural control. Prog Neurobiol 81: 45-60, 2007.

Langlet C, Leblond H, Rossignol S. Mid-lumbar segments are needed for the expression of locomotion in chronic spinal cats. J Neurophysiol 93: 2474-2488, 2005.

Macpherson JM, Inglis JT. Stance and balance following bilateral labyrinthectomy. Prog Brain Res 97: 219-228, 1993.

Massion J. Postural control system. Curr Opin Neurobiol 4: 877-887, 1994.

Massion J, Alexandrov A, Frolov A. Why and how are posture and movement coordinated? Prog Brain Res 143: 13-27, 2004.

Mori S. Integration of posture and locomotion in acute decerebrate cats and in awake, freely moving cats. Prog Neurobiol 28: 161-195, 1987.

Mori F, Nakajima K, Tachibana A, Takasu C, Mori M, Tsujimoto T, Tsukada H, Mori S. Reactive and anticipatory control of posture and bipedal locomotion in a nonhuman primate. Prog Brain Res 143: 191-198, 2004.

Nieuwkoop PD, Faber J. Normal table of Xenopus laevis (DAUDIN). A systematical and chronological survey of the development from the fertilized egg till the end. North Holland Publishing Co, Amsterdam, 1956.

Peters SE. Properties of twitch motor units of the ankle extensor muscles in the bullfrog Rana catesbeiana. J Morphol 221: 121-131, 2005. 


\section{Rauscent A, Le Ray D, Cabirol-Pol M-J, Sillar KT, Simmers J, Combes D.} Development and neuromodulation of spinal locomotor networks in the metamorphosing frog. J Physiol (Paris) 100: 317-327, 2007.

Ritter D. Epaxial muscle function during locomotion in a lizard (Varanus salvator) and the proposal of a key innovation in the vertebrate axial musculoskeletal system. $J$ Exp Biol 198: 2477-2490, 1995.

Ritter D. Axial muscle function during lizard locomotion. J Exp Biol 199: 2499-2510, 1996.

Roberts A, Soffe SR, Wolf ES, Yoshida M, Zhao FY. Central circuits controlling locomotion in young frog tadpoles. Ann NY Acad Sci 860: 19-34, 1998.

Rossignol S, Dubuc R, Gossard JP. Dynamic sensorimotor interactions in locomotion. Physiol Rev 86: 89-154, 2006.

Schotland JL, Tresch MC. Segmental and propriospinal projection systems of frog lumbar interneurons. Exp Brain Res 116: 283-298, 1997.

Shi YB. Amphibian metamorphosis: from morphology to molecular biology. New York: Wiley-Liss Inc., 2000.

Soffe SR, Roberts A. Tonic and phasic synaptic input to spinal cord motoneurons during fictive locomotion in frog embryos. J Neurophysiol 48: 1279-1288, 1982.

Stuart DG. Integration of posture and movement: Contributiuons of Sherrington, Hess, and Bernstein. Hum Mov Sci 24: 621-643, 2005.

Takakusaki K, Oohinanta-Sugimoto J, Saitoh K, Habaguchi T. Role of basal ganglia-brainstem systems in the control of postural muscle tone and locomotion. Prog Brain Res 143: 231-237, 2004.

Tokizane T, Murao M, Ogata T, Kondo T. Electromyographic studies on tonic neck, lumbar and labyrinthine reflexes in normal persons. Jpn J Physiol 2: 130-146, 1951.

Tunstall MJ, Roberts A. A longitudinal gradient of synaptic drive in the spinal cord of Xenopus embryos and its role in coordination of swimming. J Physiol 474: 393-405, 1994.

Vallois HV. Les transformations de la musculature de l'épisome chez les Vertébrés. Paris, Doin, 1922. 
Vinay L, Brocard F, Clarac F, Norreel JC, Pearlstein E, Pflieger JF. Develoment of posture and locomotion: an interplay of endogenously generated activities and neurotrophic actions by descending pathways. Brain Res Rev 40: 118-129, 2002.

Zomlefer MR, Provencher J, Blanchette G, Rossignol S. Electromyographic study of lumbar back muscles during locomotion in acute high decerebrate and low spinal cats. Brain Res 290: 249-260, 1984. 


\section{FIGURE LEGENDS}

Figure 1: Metamorphosis-induced modifications in bilateral axial/dorsal muscle coordination in free swimming Xenopus laevis. A: Axial muscle coordination in a pre-metamorphic tadpole (stage 55, left). Raw EMG recordings (middle, top two traces) and corresponding integrated traces (bottom two traces) from left (L) and right (R) axial/dorsal (ad) myomeres at the fifth spinal segment level. Right: Circular plot of onset phases of left versus right axial muscle bursts showing tendency for alternation (mean vector direction $\sim 180^{\circ}$ ). B: Coordination of the equivalent muscles in a late-metamorphosing tadpole (stage 60). Same panel arrangement as in A. Note that depending on the mode of locomotion (shaded area $\mathbf{1}$ and timebase expansion below: larval-like body undulations; shaded area $\mathbf{2}$ and timebase expansion below: adult-like leg kicking) the dorsal muscle coordination switched from alternation to synchrony as indicated by the change in mean vector direction from $\sim 180^{\circ}$ to $\sim 0^{\circ}$ in polar plots at right. C: Coordination of bilateral dorsalis trunci muscle activity in a post-metamorphic froglet (stage 66). Same panel arrangement as in A. Synchronous left-right back muscle bursts occur during locomotion that is now exclusively limbbased.

Figure 2: Kinematics of free swimming in juvenile adult Xenopus. A: Rectilinear forward swimming in stage 66 froglets. A1: Three sample images during a complete cycle of hindlimb extension-flexion with corresponding stick diagrams of back and hindlimb position reconstructed from markers redrawn on the images. A2: Upper traces: Excursions of right and left knee and ankle angles throughout a $5.5 \mathrm{~s}$ forwardswimming episode (dotted line represents body axis; arrows indicate the direction of 
knee excursion during hindlimb extension). Vertical scale bar: $100^{\circ}$ ). Bottom traces: Simultaneous EMG recordings from ankle extensor plantaris longus of the right (Rpl) and left (L-pl) hindlimbs. Note the strict bilateral synchrony of the muscle bursts. B: Non-rectilinear swimming. Same arrangement as in A. Note the irregularity and dissociation of limb trajectories (B1), knee and ankle excursions (B2, upper), and between plantaris longus activity on the two sides (B2, lower).

Figure 3: Coordination between bilateral back and hindlimb motor activity during real and fictive swimming in juveniles. A-C: Analysis of rectilinear forward swimming in vivo. A: Locality of recorded muscles in intact freely behaving animals. Left and right plantaris longus (ankle extensor) and dorsalis trunci (back muscle) were recorded simultaneously. B: EMG recordings (upper traces) of plantaris longus ( $\mathrm{R} \mathrm{pl,} \mathrm{L} \mathrm{pl)} \mathrm{and} \mathrm{dorsalis} \mathrm{trunci} \mathrm{(L} \mathrm{dt,} \mathrm{R} \mathrm{dt}$ ) with integrated transforms (lower traces) during free swimming. Dotted lines are positioned at the onset of plantaris longus burst activity. C: Circular phase plots of left versus right back (L/R dt) and hindlimb (L/R pl) muscle activity, and of back versus leg muscle activity ( $\mathrm{dt} / \mathrm{pl}$ ). Note the tendency for burst synchrony in all three phase relationships. D-F: Analysis of fictive swimming in vitro. D: Localization of the somata of motoneurons innervating left (red) and right (green) dorsalis trunci muscles in the second thoracic cord segment (Th2), and isolated brainstem/spinal cord preparation allowing suction electrode recordings from dorsalis motor axons in Th2 ventral roots, and en passant recordings from motor nerves to plantaris longus (pl) and tibialis anterioris (ta). E: Simultaneous recordings from bilateral plantaris $(\mathrm{R}$ and $\mathrm{L} \mathrm{pl}$ ) and tibialis ( $\mathrm{L}$ and $\mathrm{R}$ ta) motor nerves with the left and right ventral roots of the second thoracic segment ( $\mathrm{L}$ and $\mathrm{R}$ Th2). 
Corresponding integrated traces are shown below with dotted lines positioned at $\mathrm{L}$ and $\mathrm{R}$ plantaris longus nerve burst onsets. Such symmetrically-organized motor patterns are presumed to correspond to fictive forward swimming. F: Circular phase analysis of burst coordination in vitro, including the three relationships corresponding to the in vivo analysis in $\mathrm{C}$ (upper 3 polar plots). G: Comparison of the phase dispersions (in degrees) in these relationships in vivo (unfilled bars) and in vitro (dashed bars). CNS isolation increased the dispersion of left versus right thoracic/back phase values $(*$ : $\mathrm{p}<0.05$ ), while the distributions of the two other phase relationships remained unaltered. A-C and D-F are from two different groups of animals.

Figure 4: Effect of a complete cervico-thoracic cord transection on back and hindlimb muscle coordination. A: Drawing showing lesion location and extent, together with sample EMG recordings and associated integrated traces. B: Circular analysis of the three indicated burst relationships, corresponding to those analyzed in intact control animals (see Fig. 3). *** $(\mathrm{p}<0.001)$ above polar plots indicate differences (Mardia-Watson-Wheeler test) from control distributions (as seen in Fig. 3C). C: Histograms of phase dispersions (derived from B) in lesioned animals (filled bars) compared to intact controls (unfilled bars). *** above bars indicate significance $(\mathrm{p}<0.001)$ of differences (two-tailed unpaired t-test) from control group.

\section{Figure 5: Effect of a sagittal thoracic cord lesion on back and hindlimb motor} burst coordination in vivo and in vitro. A-D: Post-lesion analysis of muscle coordination in vivo. A: Schematic of the localization and extent of the midline cord lesion. Dots indicate sites of EMG recordings. B: Sample EMG recordings and 
corresponding integrated traces. C: Circular analysis of indicated phase relationships (same arrangement as in Fig. 3). $* * *(\mathrm{p}<0.001)$ and ns (non significant) above polar plots indicate the significance (Mardia-Watson-Wheeler test) of differences from control distributions (as seen in Fig. 3C). D: Histograms of phase dispersions around the mean vector $\mu$ for control (unfilled bars) and lesioned (filled bars corresponding to circular plots in C) groups of animals. E-H: Analysis of left/right and longitudinal motor burst coordination in vitro. Same arrangement as in A-D with additional recordings (and phase analyses) from left and right tibialis ( $\mathrm{L}$ and $\mathrm{R}$ ta) motor nerves. $* \mathrm{p}<0.05 ; * * \mathrm{p}<0.01 ; * * * \mathrm{p}<0.001$. A-D and $\mathrm{E}-\mathrm{H}$ are from two different animal groups.

Figure 6: Effect of thoraco-lumbar cord transections on back and leg muscle coordination. A-C: Complete transection. A: Sample episodes evoked by a brief mechanical stimulation (at arrowheads) of either a hindlimb ( $\left.\mathrm{h}_{\text {stim }}\right)$ or forelimb $\left(\mathrm{f}_{\text {stim }}\right)$ after a thoraco-lumbar transection, as indicated in the drawing above. Recordings at left show uncoordinated dorsal and hindlimb activity during hindlimb movement, and recordings at right show bilateral dorsalis trunci activity in the absence of limb muscle activity. B-C: Same arrangement as in Fig. 4, but note the absence of $\mathrm{L} / \mathrm{R}$ plantaris longus analysis due to the lack of organized hindlimb motor activity after such a lesion. $* * \mathrm{p}<0.01$. D-F: Hemi-transection of left cord side combined with a sagittal lesion of the three thoracic segments. D: Sample spontaneous episodes recorded during swimming (1) and in the absence of limb kicking (2). When the animal swam, the right dorsalis was rhythmically activated while the left side remained silent. In contrast, dorsalis bursts could still occur on both sides in the 
absence of locomotor activity. E: Circular analysis of left (Ldt) and right (Rdt) dorsalis bursts compared to ipsilateral plantaris ( $\mathrm{pl}$ ) activity showing the lack of correspondence between left dorsal and locomotor muscle contractions. F: The phase dispersions were significantly wider $(\mathrm{p}<0.001)$ on the left, lesioned side.

\section{Figure 7: Organization of spinal lumbar and thoracic circuitry for locomotion and} posture in pre- and post-metamorphic Xenopus. A: In the tadpole, spinal circuitry consists of a distributed chain of segmental oscillators that generate alternating leftright myotomal contractions by reciprocal inhibitory cross-cord connections. Propulsive body undulations during swimming are produced by a rostro-caudal propagation of motor activity that is coordinated by brainstem-derived gradients of neuronal excitability extending progressively down the cord. Schematic derived from earlier work on post-embryonic Xenopus larvae (Tunstall and Roberts 1994; see also Roberts et al. 1998). B: In the young adult spinal cord, the dorsal axial musculature is now controlled by weakly interacting bilateral thoracic circuitry that may be activated by oppositely-directed longitudinal influences: the first descends directly from brainstem postural centers (thin dotted arrows) to command reactive postural adjustments via appropriate back and hindlimb muscle activation; the second pathway ascends from the hindlimb locomotor command network located within the lumbar cord segments (thick medial arrows). Note that another pathway, descending from the cervical networks controlling forelimbs (thin grey medial arrows), may also constitute to some extent a direct pathway between dorsal motoneurons and a spinal locomotor network. Although the lumbar CPG requires activation by brainstem locomotor centers (unfilled lateral arrows), the lumbo-thoracic pathway enables dynamic 
postural control whereby back muscle contractions are matched proactively to ongoing locomotor movements. 


\section{TABLE LEGEND}

\section{Table 1: Effects of multiple spinal lesions in vivo.}

Circular statistics of the coordination between left and right dorsalis and plantaris longus muscles, and between back and hindlimb muscles ( $\mathrm{dt} / \mathrm{pl})$ following combined cervico-thoracic cord transection, sagittal thoracic section, and thoraco-lumbar rhizotomy. Results of the Rayleigh's uniformity test $(Z)$, the $\mathrm{V}$-test for the $0^{\circ}$ direction of the mean vector $\left(u\left(0^{\circ}\right)\right)$, and the Mardia-Watson-Wheeler test $(W)$ indicate that the three bilateral and longitudinal relationships examined were not significantly modified by these multiple lesions, although phase distribution variability decreased for $1 / \mathrm{r}$ plantaris longus and $\mathrm{dt} / \mathrm{pl}$ coupling. The control $r$ value for each relationship is indicated in brackets. 
A

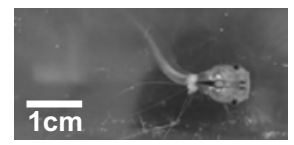

axial/dorsal

muscles

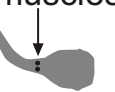

B

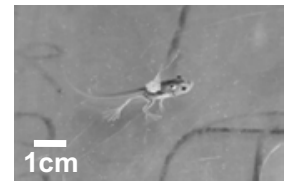

axial/dorsal muscles

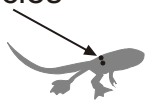

C

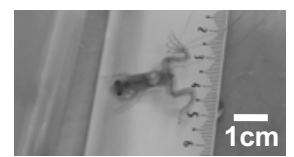

dorsal muscles (dorsalis trunci) trunci)
$L$ ad

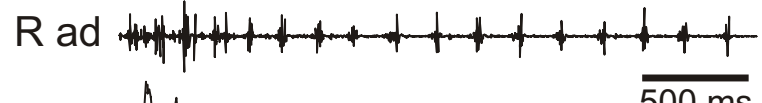

$\mathrm{L}$ ad Shoming $\overline{500 \mathrm{~ms}}$ $\mathrm{R}$ ad $M M M_{1} \sin _{1}$

(1)

$L$ ad Hullith Hall (2)

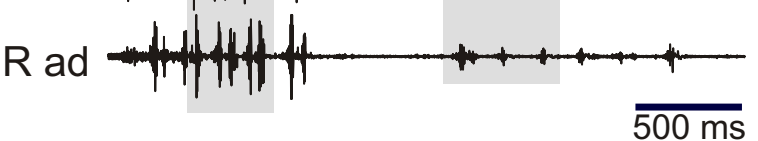

(2)

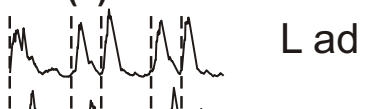
Noncher R ad

$$
1 \overline{00 \mathrm{~ms}}
$$

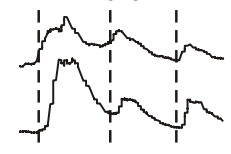

$100 \mathrm{~ms}$

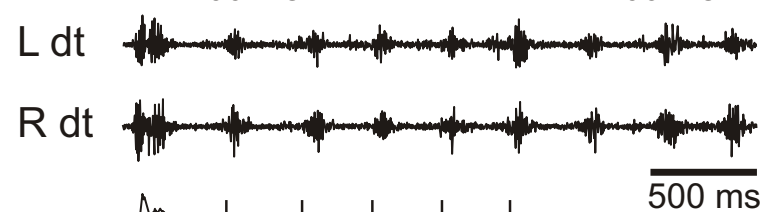

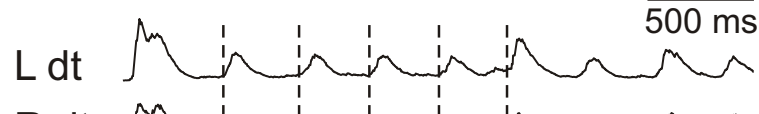

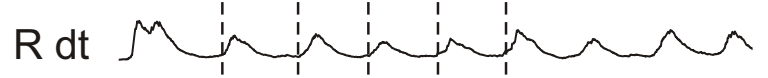

alternation

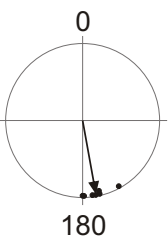

(1)

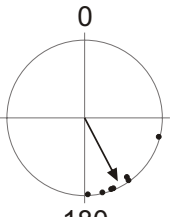

(2)
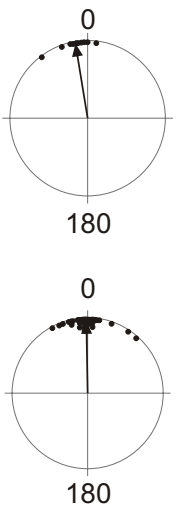

synchrony

Beyeler et al.

Figure 1 
A1
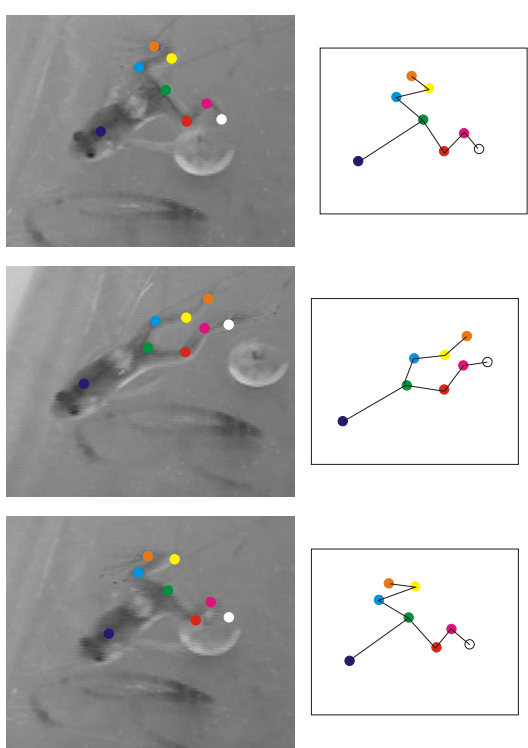

2

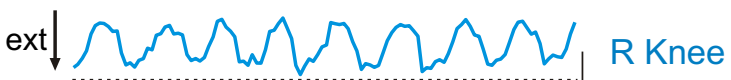

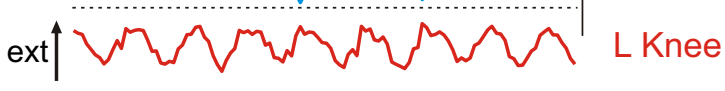

$\operatorname{ext} / M W M M M W W_{\text {R Ankle }}$

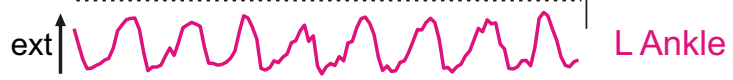

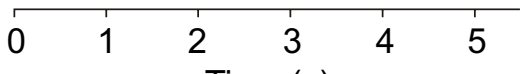

Time (s)

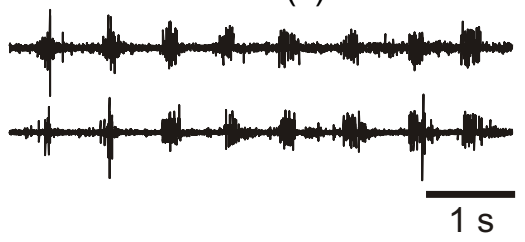

B 1
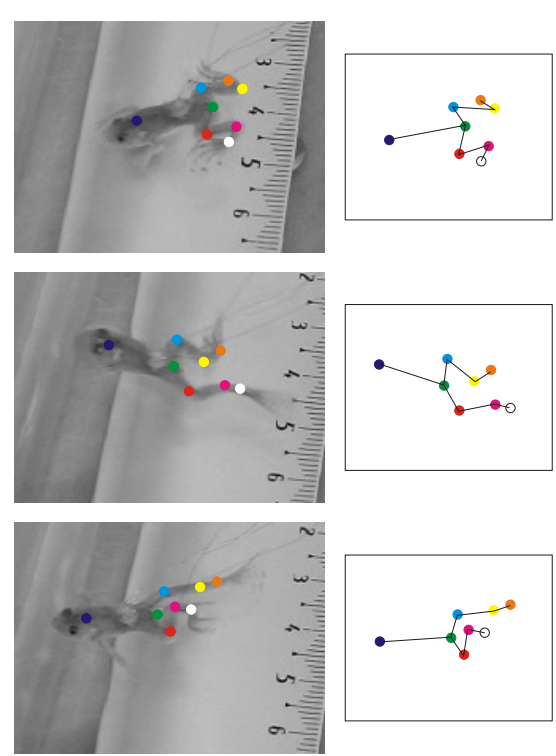

2
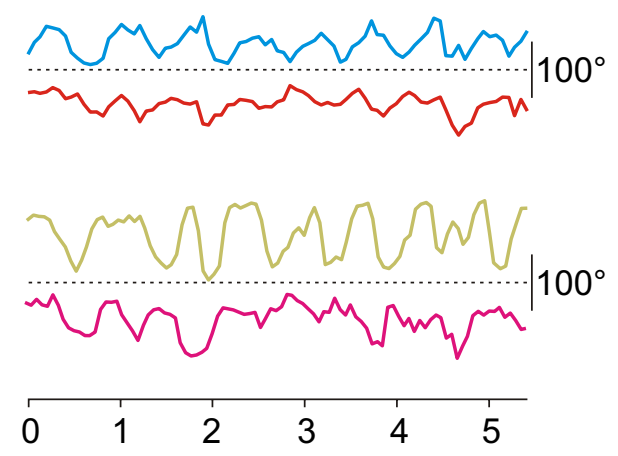
Time (s)

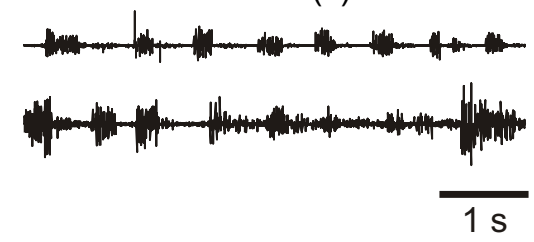

Beyeler et al.

Figure 2 
A In vivo

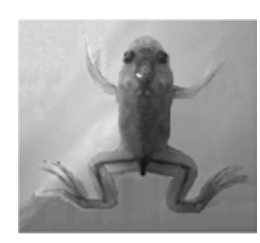

dorsalis trunci

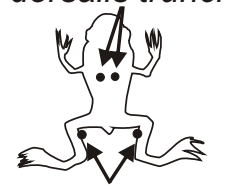

plantaris longus
D

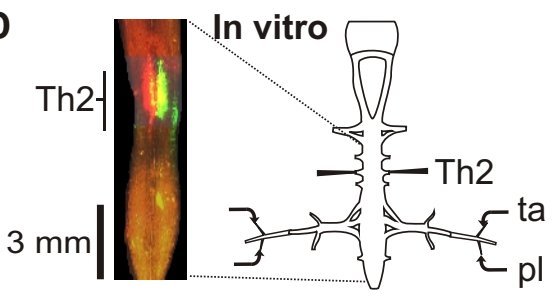

B

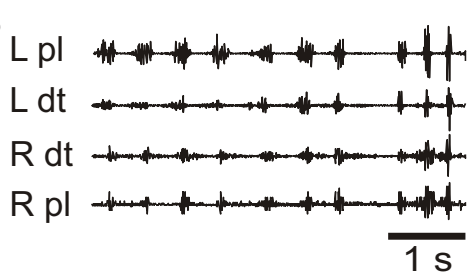

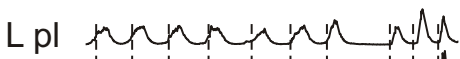

Ldt

$R d t$ r

$R \mathrm{pl}$

E

L ta

$\mathrm{L} \mathrm{pl}$

L Th2

R Th2

$\mathrm{R} \mathrm{pl}$

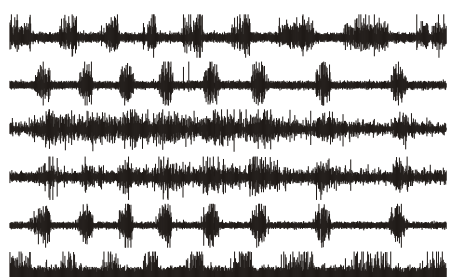

$\mathrm{R}$ ta

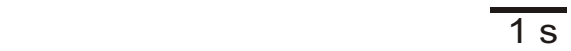

$L$ ta

$\mathrm{L} \mathrm{pl}$

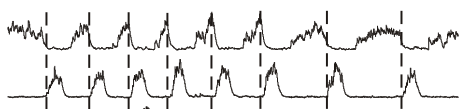

LTh2

C L/R dt L/R pl dt/pl

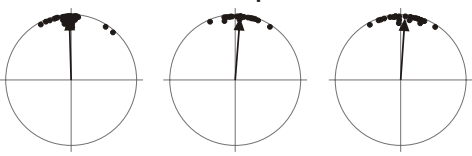

R Th2 N

$\mathrm{R} \mathrm{pl}$

$R$ ta

G In vivo / in vitro

F L/RTh2 L/R pl Th2/pl

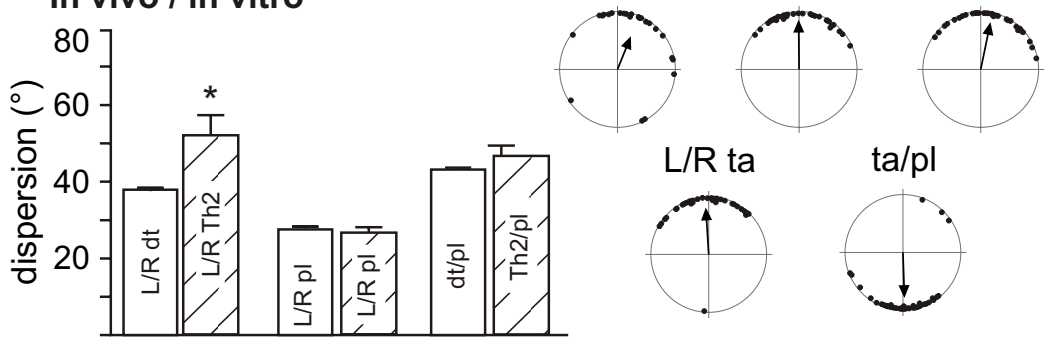

Beyeler et al.

Figure 3 
A In vivo

$$
\text { ? }
$$

L pl
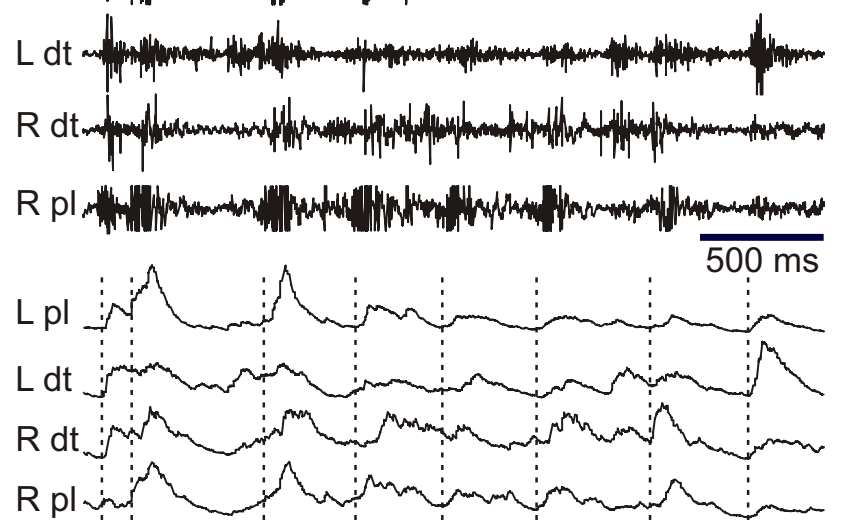

B

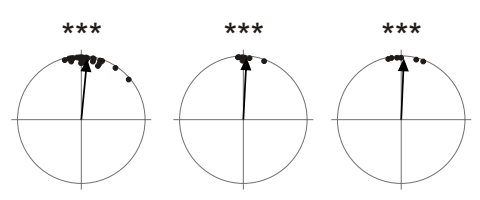

L/R dt L/R pl $\quad d t / p l$

\section{C}

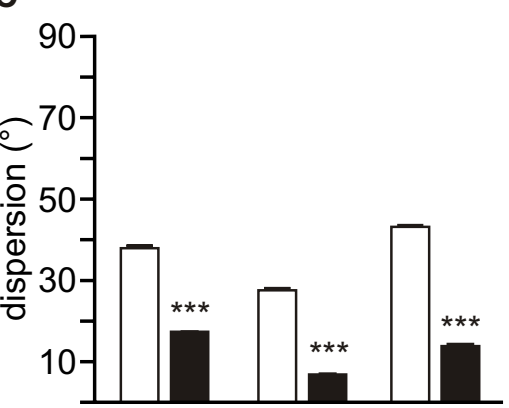

Beyeler et al.

Figure 4 


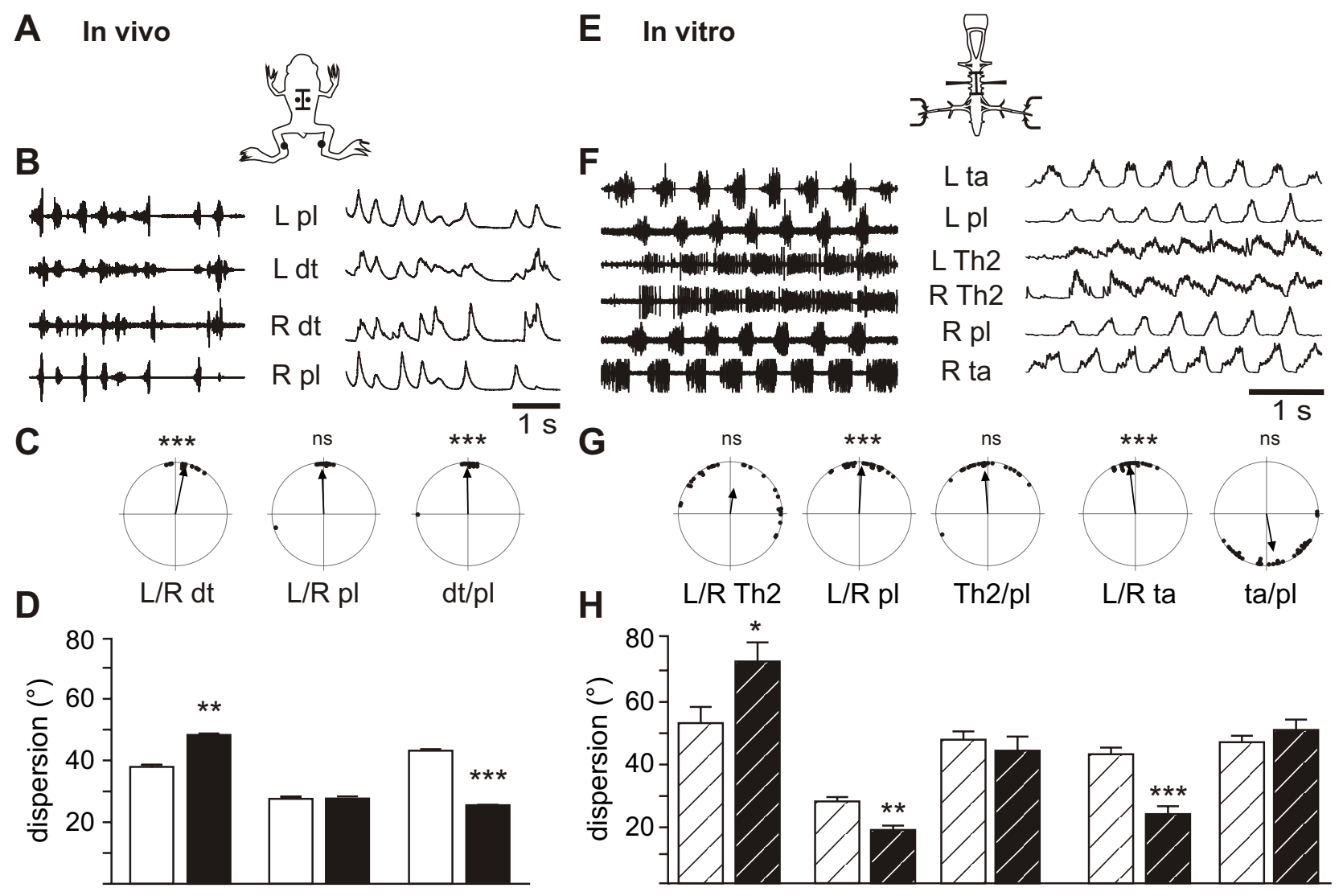

Beyeler et al.

Figure 5 
A In vivo

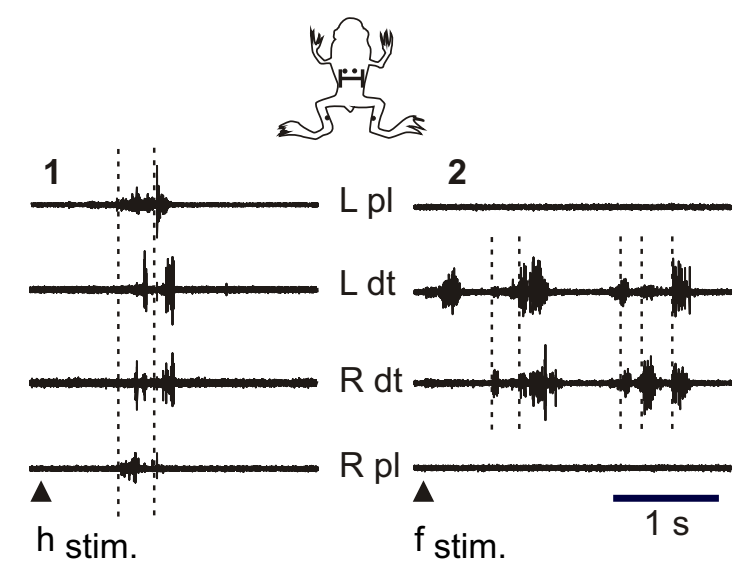

D

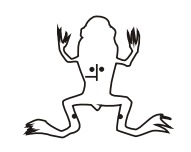

1

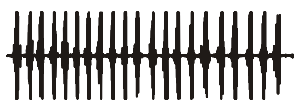

Him

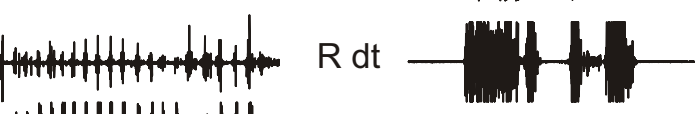

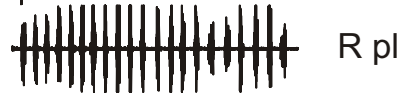

2
B

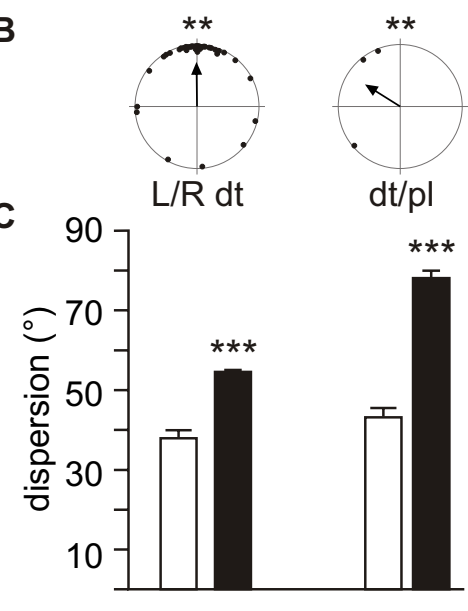

E

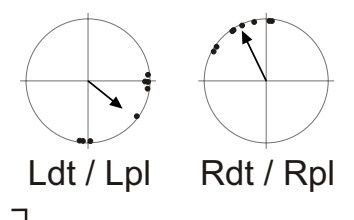

F $\quad 90 \mathrm{Ldt} / \mathrm{Lpl} \quad \mathrm{Rdt} / \mathrm{Rpl}$

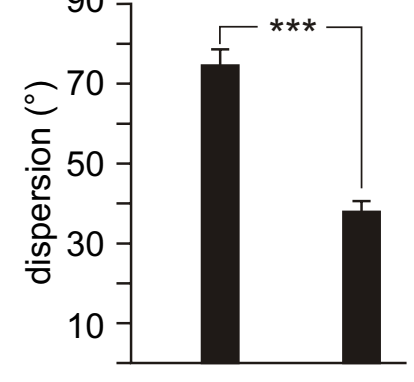

Beyeler et al.

Figure 6 


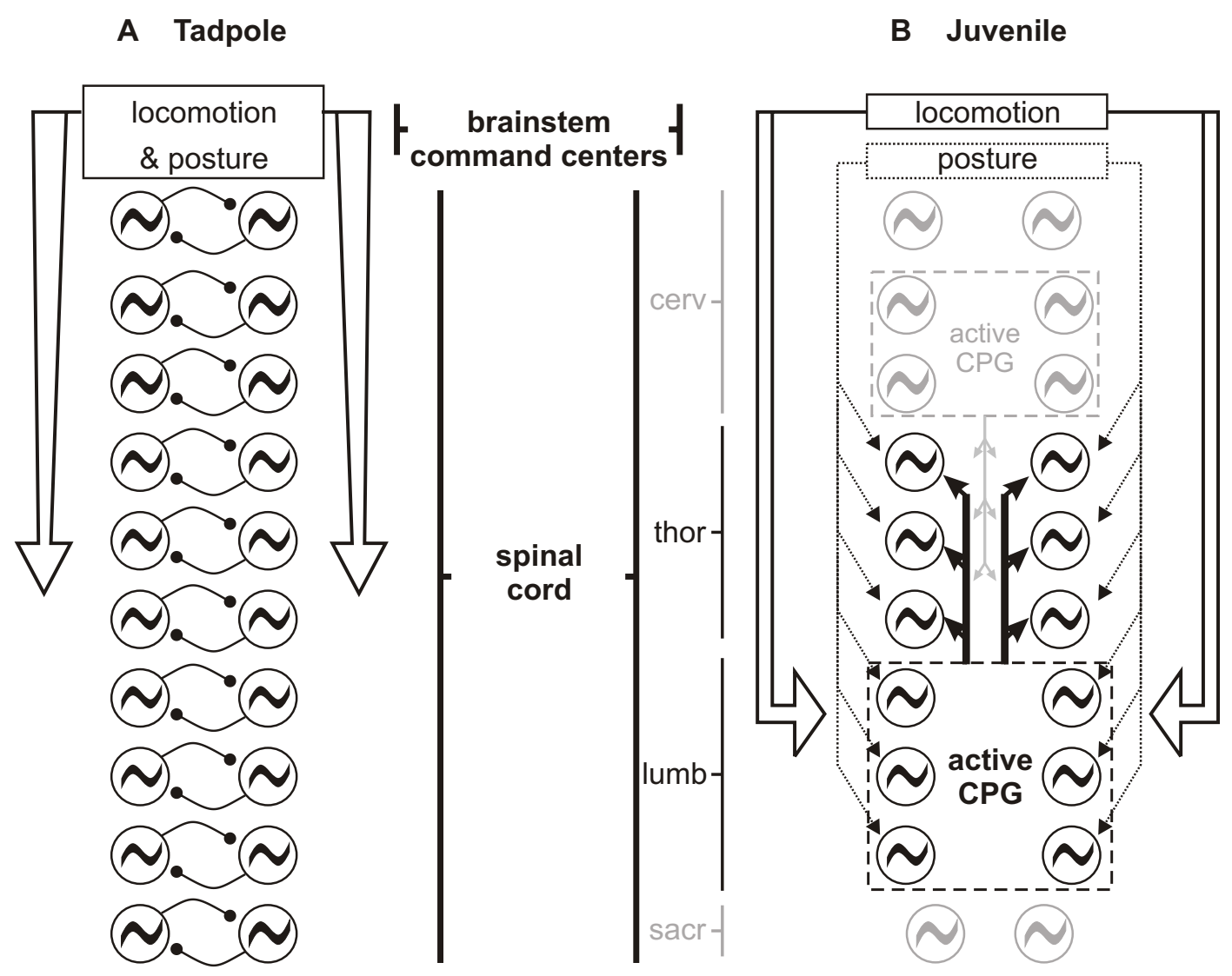

Beyeler et al. Figure 7 
Beyeler et al.

Table 1

\begin{tabular}{|c|cc|cc|cc|cc|}
\cline { 2 - 9 } \multicolumn{1}{c|}{} & $\mu\left(^{\circ}\right)$ & $r$ & $Z$ & $\mathrm{p}$ & $u\left(\mathrm{O}^{\circ}\right)$ & $\mathrm{p}$ & $W$ & $\mathrm{p}$ \\
\hline $\begin{array}{c}\text { left/right } \\
\text { dorsalis trunci }\end{array}$ & 10.40 & $\begin{array}{l}0.89 \\
(0,65)\end{array}$ & 7.99 & $<0,001$ & 3.93 & $<0,001$ & 3.70 & $>0,05$ \\
\hline $\begin{array}{c}\text { left/right } \\
\text { plantaris longus }\end{array}$ & 3.36 & $\begin{array}{l}0.99 \\
(0,80)\end{array}$ & 10.81 & $<0,001$ & 4.64 & $<0,001$ & 14.53 & $<0,001$ \\
\hline$d t / p /$ & 357.32 & $\begin{array}{c}0.88 \\
(0,59)\end{array}$ & 16.95 & $<0,001$ & 5.82 & $<0,001$ & 9.83 & $<0,01$ \\
& & & & & & & \\
\hline
\end{tabular}

